\title{
Cytokine-induced killer cells co-cultured with complete tumor antigen-loaded dendritic cells, have enhanced selective cytotoxicity on carboplatin-resistant retinoblastoma cells
}

\author{
YA-FENG WANG ${ }^{1,2}$, PATRICIA E. KUNDA ${ }^{3}$, JIAN-WEI LIN ${ }^{3}$, HAN WANG $^{3}$, \\ XUE-MEI CHEN ${ }^{3}$, QIU-LING LIU $^{2}$ and TAO LIU $^{3}$ \\ ${ }^{1}$ Graduate Division, Xinxiang Medical University, Xinxiang, He'nan 453003; ${ }^{2}$ Department of Pediatrics, \\ The General Hospital of the Chinese People's Armed Police Forces, Beijing 100039; \\ ${ }^{3}$ Poten Biomedical Institute for Cancer Immunotherapy, Shenzhen, Guangdong 518000, P.R. China
}

Received December 11, 2012; Accepted January 30, 2013

DOI: 10.3892/or.2013.2315

\begin{abstract}
Retinoblastoma (RB) is a challenging disease that affects mostly young children. Chemical therapy has been shown to have limitations during clinical practice, principally because of the ability of RB to become resistant to the treatment. Nevertheless, chemotherapy is still the main treatment for RB, and immunotherapy has become a promising treatment for most solid tumors with fewer side effects than traditional therapies. In this study, we explored the antitumor effects of cytokine-induced killer (CIK) cells co-cultured with dendritic cells (DCs) pulsed with complete tumor antigens (DC-Ag). Cytotoxicity and specificity were evaluated on an RB cell line (RB-Y79), on a human normal retina cell line (hTERT-RPE1) and a carboplatin-resistant RB cell line. Our results showed that CIK differentiation and cytotoxicity were enhanced by co-culturing CIKs with DC-Ag. Moreover, the co-culture improved the CIK proliferation rate by increasing IL- 6 and decreasing IL-10 levels in the culture medium. Furthermore, the use of DC-Ag-CIK cells had little effect on normal retinal cells but high cytotoxicity on RB cells even on carboplatinresistant retinoblastoma cells. This is the first study showing that DC cells pulsed with the complete tumor antigen improve proliferation, differentiation and cytotoxic activity of CIKs specific not only for RB but also for the chemotherapy-resistant form of the malady. Thus highly efficient immunotherapy based on DC-Ag-CIK cells may be a potential effective and
\end{abstract}

Correspondence to: Professor Qiu-Ling Liu, Department of Pediatrics, The General Hospital of the Chinese People's Armed Police Forces, Beijing 100039, P.R. China

E-mail:wj670@vip.sina.com

Dr Tao Liu, Poten Biomedical Institute for Cancer Immunotherapy, Shenzhen, Guangdong 518000, P.R. China

E-mail: tao.liu2008@gmail.com

Key words: dendritic cells, cytokine-induced killer cells, retinoblastoma, carboplatin resistant, immunotherapy, chemical therapy safe mean of treating RB especially to patients where traditional chemical therapy has failed.

\section{Introduction}

Retinoblastoma (RB) is the most common intraocular malignancy in children. Worldwide, the incidence of RB is $\sim 1$ in 15,000 , with nearly 9,000 new cases of RB reported each year (1). In China, most children with RB are in the late-stage of the disease because of delayed/late diagnosis. The morbidity and mortality are significantly higher than in developed countries $(2,3)$. Patients with RB are typically children $<5$ years old and $>89 \%$ of patients are $<3$ years old at the onset of RB; some present symptoms at birth (4). At present, the main treatments are systemic chemotherapy combined with local treatment (1). Although the efficacy of chemotherapy has recently improved, the long-term systemic chemotherapy can cause severe side effects, such as bone marrow suppression, ototoxicity, renal toxicity and other adverse reactions that affect the child's quality of life $(5,6)$. These side effects are particularly serious because children are in an active phase of development during the treatment. Other disadvantages include the induction of multidrug resistance induced by the high-dose chemotherapy used to treat $\operatorname{RB}(7,8)$. Therefore, finding a new method to treat $\mathrm{RB}$ effectively has become a priority. Biological treatments (and in special adoptive immunotherapy) have been shown to have great potential as an adjunct treatment to control the disease. Adoptive immunotherapy uses the natural ability of the immune system to recognize and eliminate continuously arising transformed cells. This approach can be efficiently employed for the eradication of residual cancer cells and prevention or delay of tumor relapse (9). An example is the case of solid tumors, where antigen-specific immunotherapy has emerged as a promising approach (10-12). One of the key players in mediating immune response are the dendritic cells (DCs) as they are specialized in priming naive helper and cytotoxic T-lymphocytes and directly trigger natural killer (NK) cell function (13). In addition, DCs can be loaded with antigens (DC-Ag) that may increase the DC's specificity. This in turn enhances the targeting of killing cancer cells (14-16). Functional T-lymphocytes can be gener- 
ated in vitro from peripheral blood mononuclear cells (PBMCs) by using specific media formulations. A sub-population of the T-lymphocytes, differentiated in vitro by addition of specific cytokines to the media culture is the cytokine-induced killer (CIK) cells. The CIK cells are major histocompatibility complex (MHC)-unrestricted with the characteristic $\mathrm{CD} 3{ }^{+} \mathrm{CD} 56^{+}$phenotype and they have been shown, in vitro and in medical practice, to be efficient killers of tumor cells (9,17-19). These CIK cells with the ability to attack tumor cells are expressed on the cell surface of CD3/CD56. Some studies have shown that CIKs co-cultured with DCs (DC-CIKs) can have better antitumor activity on a variety of cancers (20-22). However, until now DC-CIKs have not been reported to exhibit cytotoxicity towards RB cells.

There are some questions about the ability of the antigens obtained from cancer cell line lysates to generate enough cancer cell targeting specificity to allow for DC-CIKs to be used extensively for clinical treatment instead of a personalized therapy. However, the ability of the antigen (Ag) loaded DC-CIK co-culture (DC-Ag-CIK) to transactivate one another via the reciprocal production of cytokines could promote more tumor killing activity in vitro. This enhanced tumor killing activity could overcome the limitation of not using a personalized therapy. In advanced RB cases, chemotherapy is the main available treatment that offers a chance of rescuing the eyeballs. However, the drugs used in this treatment have a negative effect on humoral and cellular immunity and the damage to the immune system is related to both the dose and the duration of administration (23,24). Clearly then an effective immunotherapy treatment against RB would be advantageous. Thus, we evaluated the antitumor immune responses of immunotherapy on RB cells in vitro and provide evidence for a new method for treating RB. We used RB antigen pulsed DCs co-cultured with CIK cells to investigate the cytotoxic effect on the RB cell line RB-Y79. Our results showed that the Ag pulsed DC cells promote cell proliferation and increase the CIK differentiation in vitro by regulating the secretion of IL-6 and decreasing IL-10 cytokine levels. Also we show that Ag pulsed DCs specifically enhanced the CIK cytotoxicity on RB cells. Importantly, this is the first demonstration of the ability of DC-Ag-CIKs to kill RB cells and carboplatin resistance $\mathrm{RB}$ cells. Because it is specific for RB with no cytotoxic effect on normal retina cells, this immunotherapeutic approach could represent a safe, less toxic and effective treatment for RB.

\section{Materials and methods}

Cell lines and reagents. Human retinoblastoma cell line RB-Y79 was purchased from American Type Culture Collection (ATCC, Rockville, MD, USA), human retinal pigment epithelium cell line hTERT-RPE1 was purchased from JENNIO Biological Co. (Guangzhou, China). All the cells were cultured at $37^{\circ} \mathrm{C}$ in a humidified incubator under 5\% $\mathrm{CO}_{2}$ in RPMI-1640 (Gibco, USA) with $10 \%$ fetal bovine serum (FBS) (Gibco, Australia) and $1 \%$ penicillin-streptomycin solution (Gibco, USA). All anti-human CD3-FITC, CD8-APC, CD56-PE, CD80-PE, CD83-APC, CD86-FITC antibodies were obtained from BD Co. (BD, USA). Cytokines recombinant mutant human tumor necrosis factor- $\alpha$ (TNF- $\alpha$ ), recombinant human interferon- $\gamma$ (IFN- $\gamma$ ), recombinant human interleukin-2 (IL-2), recom- binant human granulocyte/macrophage colony-stimulating factor (GM-CSF), recombinant human interleukin-4 (IL-4) and CD3 monoclonal antibody were purchased from Peprotech (Rocky Hill, NJ, USA). Propidium iodide (PI) and 5-carboxy-fluorescein diacetate succinimidyl ester (CFSE) were purchased from Beyotime Institute of Biotechnology (Shanghai, China), Carboplatin was purchased from Qilu Pharmaceutical Co. (Ji'nan, Shandong, China).

Generation of dendritic cells and CIK cells. Blood was freshly drawn from healthy volunteers according to our protocol accepted by the local ethics committee (LEC). PBMCs were isolated by Ficoll/Hypaque density gradient centrifugation, according to standard procedures (25). PBMCs at a density of $5 \times 10^{6}$ cells $/ \mathrm{ml}$ were allowed to adhere in Alys-505 complete culture medium (Alys-505 medium supplemented with $0.5 \%$ auto-plasma and $1 \%$ penicillin/streptomycin) for $3 \mathrm{~h}$ at $37^{\circ} \mathrm{C}$ in a humidified $5 \%$ $\mathrm{CO}_{2}$ incubator. The adherent PBMCs were cultured in Alys-505 complete medium with $500 \mathrm{U} / \mathrm{ml}$ GM-CSF and $1000 \mathrm{U} / \mathrm{ml} \mathrm{IL-4}$ to generate DCs. The medium was changed every 3 days. In the experiment where TNF- $\alpha$ was tested, $1000 \mathrm{U} / \mathrm{ml}$ of TNF- $\alpha$ was added to the media on day 6 . The non-adherent PBMCs were cultured in Alys-505 complete medium containing $1000 \mathrm{IU} / \mathrm{ml}$ IFN- $\gamma, 200 \mathrm{ng} / \mathrm{ml} \mathrm{CD} 3$ monoclonal antibody and $250 \mathrm{U} / \mathrm{ml} \mathrm{IL-2}$ for generating CIK cells. After day 7 in culture, CIK cells were sub-cultured in fresh Alys-505 complete culture medium with $200 \mathrm{U} / \mathrm{ml}$ IL-2 every 3 days.

Tumor lysates and pulsing DCs. RB-Y79 tumor lysates were generated by three rapid freeze-thaw cycles. Briefly, confluent cultures of RB-Y79 were detached by incubation with trypsinEDTA $0.05 \%$ for $5 \mathrm{~min}$, washed in PBS and resuspended in PBS at a density of $1.5 \times 10^{7}$ cells $/ \mathrm{ml}$. The cells in suspension were frozen in liquid nitrogen and disrupted by three freezethaw cycles. The cell lysis was clarified by centrifugation for $10 \mathrm{~min}$ at $600 \mathrm{x} \mathrm{g}$. The supernatant was then collected and stored at $-80^{\circ} \mathrm{C}$. DCs were incubated in the presence of the tumor lysates from the day 3 of DC maturation.

CIK and DC cells co-culture and cells proliferation assay. CIK cells were co-cultured on day 7 with autologous 7-day DC or DC-Ag in a 3:1 ratio. All the groups were cultured at $37^{\circ} \mathrm{C}$ in a humidified $5 \% \mathrm{CO}_{2}$ incubator and sub-cultured every 3 days in Alys-505 complete culture medium with $200 \mathrm{U} / \mathrm{ml}$ IL-2. Cell number was counted from day 7 to 15 in culture using Counter Star with the software Automated Cell Counter.

Flow cytometry. DCs were phenotyped with a panel of antibodies: CD80-PE, CD83-APC and CD86-FITC. CIK cells were phenotyped with antibodies against CD3-FITC, CD8-APC and CD56-PE. A mouse IgG (PE/FITC/APC) (BD) was used as a negative control in all the assays. Briefly, $1 \times 10^{6}$ cells were incubated with the corresponding antibodies at $4^{\circ} \mathrm{C}$ for $15 \mathrm{~min}$ and then washed with PBS. A total of 10,000 cells were measured and analysed using CellQuest Pro software. Dual-color flow cytometric analysis was performed on a BD FACSCalibur.

Quantitation of cytokine production by DC and CIK cells. On day 15, supernatants from all experimental groups were collected. IL-6 and IL-10 levels in the media culture were 
quantitatively measure by using a Cytometric Bead Array Human Th1/Th2 Cytokine kit II (BD) according to the manufacturer's instructions.

Cytotoxicity assay. CIK cells, DC-CIK cells and DC-Ag-CIK cells were harvest on day 15 and the cytotoxic activity was measured on target cells, either RB-Y79 or hTERT-RPE1 cells. Briefly, RB-Y79 cells and hTERT-RPE1 cells were suspended in PBS at a density of $1 \times 10^{5}$ cells $/ \mathrm{ml}$ and stained with $2 \mu \mathrm{mol} / 1$ CFSE for $10 \mathrm{~min}$ at $37^{\circ} \mathrm{C}$. After washing 3 times with RPMI-1640 medium containing 10\% FBS, the CFSE-labeled RB-Y79 cells or hTERT-RPE1 cells were co-cultured with CIK cells, DC-CIK cells, DC-Ag-CIK cells, respectively. A 10:1, 20:1 and 40:1 effector:target cell ratio was used. RB-Y79 or hTERT-RPE1 cells were cultured with media from the respective effector cell group and they were used as control for the natural death percentage. After culturing for $24 \mathrm{~h}$, the cells from all groups were collected and washed twice with PBS and then incubated with PI $(1 \mu \mathrm{g} / \mathrm{ml})$ at room temperature for $10 \mathrm{~min}$. Life and dead cells were analysed using dual-color flow cytometric analysis performed on a BD FACSCalibur. Each group was tested in triplicate.

For testing the effect of the media on CIK cytotoxicity, $1 \times 10^{5}$ RB-Y79 cells were co-cultured with $1 \times 10^{6}$ CIK cells using conditioned media from CIK cells, DC medium and DC-Ag medium. The cytotoxicity was analysed in triplicate as desribed above. In all experiments the cytotoxic activity was defined as the percentage of dead cells after $24 \mathrm{~h}$ of treatment less the natural death percentage of the respective cell type.

Cytotoxicity assay on $R B$-resistant cells $(R B-R)$. RB-Y79 cells in logarithmic phase were incubated with $40 \mu \mathrm{g} / \mathrm{ml}$ at $37^{\circ} \mathrm{C}$ in a humidified $5 \% \mathrm{CO}_{2}$ incubator for $2 \mathrm{~h}$. After centrifugation and washing, the medium containing the drug was discarded. Cells were cultured then in complete culture medium (RPMI $+10 \%$ FBS and $1 \%$ penicillin/streptomycin). Once the culture growth into the logarithmic phase again the Carboplatin treatment was repeated. The same procedure was repeated for several months until generating a stable resistant cell line at $40 \mu \mathrm{g} / \mathrm{ml}$ carboplatin.

For testing RB-R cell resistance to carboplatin, RB-Y79 and RB-R cells were cultured in the presence of 10, 20, 40, $50,60,70,80,90$ or $100 \mu \mathrm{g} / \mathrm{ml}$ carboplatin respectively. After $24 \mathrm{~h}$, cell numbers were counted in the culture using Counter Star with the Automated Cell Counter software. In addition, $1 \times 10^{5}$ RB-Y79 and RB-R cells were cultured with carboplatin at $40 \mu \mathrm{g} / \mathrm{ml}$, respectively. The cytotoxicity was analysed as described above.

For the cytotoxicity assay, CIK, DC-CIK cells and DC-Ag-CIK cells were harvest on day 15 and co-cultured with RB-Y79 and RB-R cells in a ratio 20:1 in the presence or absence of $40 \mu \mathrm{g} / \mathrm{ml}$ carboplatin. After culturing for $24 \mathrm{~h}$, the cells from all groups were collected and washed twice with PBS and then stained with $2 \mu \mathrm{mol} / 1 \mathrm{CFSE}$ and PI $1 \mu \mathrm{g} /$ $\mathrm{ml}$. Viable and dead cells were analysed using dual-color flow cytometric analysis performed on a BD FACSCalibur. Each group was tested in triplicate.

For every experiment the cytotoxic activity was defined as the percentage of dead cells after $24 \mathrm{~h}$ of treatment less the percentage of natural death of the respective cell type.

\section{Results}

Morphological characterization and phenotypical analysis of DC maturation in vitro. In this study mature and active DCs were generated in vitro from peripheral blood mononuclear cells (PBMCs) taken from a healthy volunteer. Cell differentiation in culture was followed over time by checking cell morphology. The mature phenotype was recognized by analyzing the expression of the clusters of differentiation (CD80, CD83, CD86) in the cell membrane using flow cytometry (FCM) (Fig. 1).

Freshly isolated PBMCs were spherical and small, the cell surface was smooth and no protrusions were observed by light microscopy (Fig. 1A, left image). After culturing for 3 days in the presence of cytokine-enriched media, the adherent cells became larger and oval. On day 7, cells lost the ability to adhere to the plastic and started to grow in suspension. An additional morphological feature of these cells was the increment in size, irregular shaped nuclei and the formation of numerous dendritic-like actin processes emerging from the cell body (Fig. 1A, middle and right images) depicting the typical DC morphology corresponding to a mature phenotype. As expected, this change in morphology was accompanied by an increase in the cell surface expression of the CDs (Fig. 1B). After 7 days in culture, FCM assay detected values for the CDs significantly higher compared with the cells on day 3

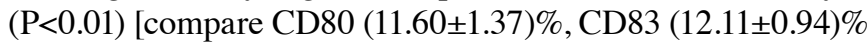
and CD86 $(15.28 \pm 0.35) \%$ on day 3 with CD80 (46.65 \pm 1.26$) \%$, CD83 (26.14 \pm 1.16$) \%$ and CD86 (31.05 \pm 1.12$) \%$ on day 7]. The results showed that DCs developed into the mature phenotype after 7 days (Fig. 1C).

To test the effect of tumor antigen on DC differentiation and maturation in vitro, tumor lysed from RB-Y79 cells (Ag) was added to the DCs from day 3 in culture. On day 7, DC-Ag cells were harvested and the same CDs as above were analysed by FCM. As shown in Fig. 1B and D (top panels), the DC-Ag cells expressed higher levels of CD80 and CD86 compared with DC cultured in cytokine enriched media alone (without $\mathrm{Ag})(\mathrm{P}<0.01)$. However, no significant differences were found for CD83 levels.

Because TNF- $\alpha$ have a positive effect on DC differentiation and maturation (26), we tested whether the antigen had a co-stimulatory effect after 1 day of treatment with TNF- $\alpha$ on DC maturation. To examine this hypothesis we analysed CD expression levels on cell surface by FCM (Fig. 1D, bottom panels). The results showed that the addition of TNF- $\alpha$ to the culture medium on day 6 increased significantly CD80, CD83 and CD86 expressions compared to DC without TNF- $\alpha(\mathrm{P}<0.01)$, with more obvious differences in the expression level of CD83. TNF- $\alpha$ had a similar effect on DC-Ag cells (Fig. 1E) $(\mathrm{P}<0.05)$.

Overall, our findings showed that the antigen could upregulate the expression of the CDs that act as co-stimulatory molecules promoting DC differentiation. In addition, TNF- $\alpha$ had a strong effect on the overall maturation of DCs.

Because TNF- $\alpha$ favored DC differentiation in vitro, the following experiments were performed in the presence of TNF- $\alpha$. Thus, we can discriminate the effects on DC function attributable to $\mathrm{Ag}$ that are independent of maturation.

Morphologic characterization and phenotypic analysis of CIK maturation in vitro. CIK cells are a sub-population of 
A
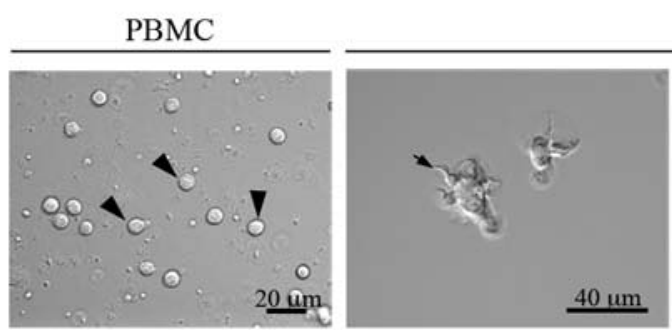

DC 7 Days in vitro

B

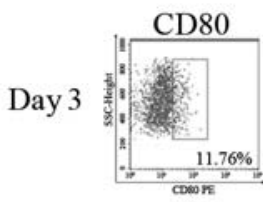

CD83

CD86
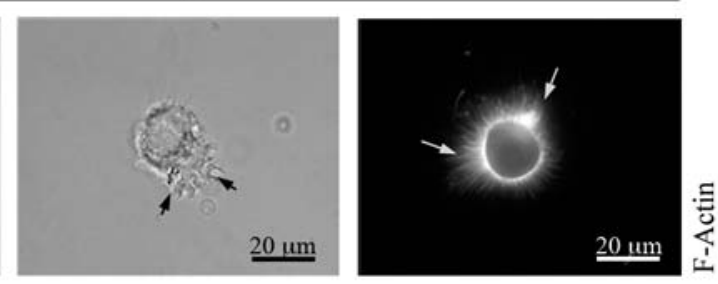

C

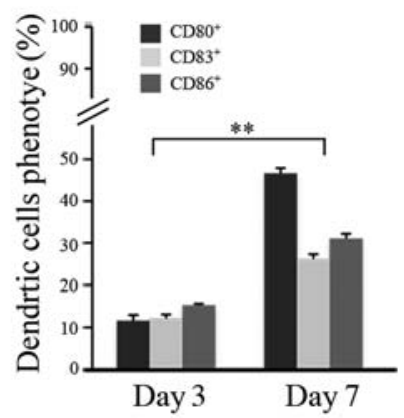

D
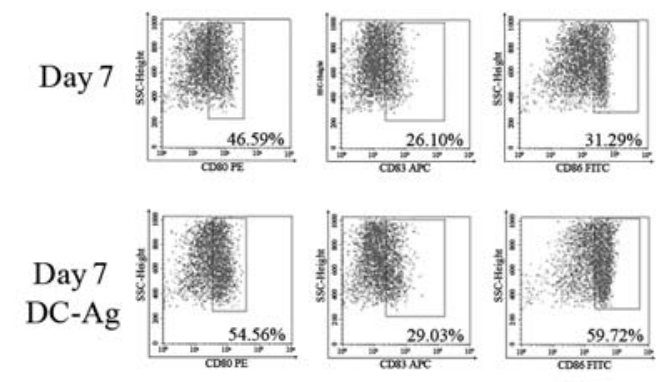

$\mathrm{E}$

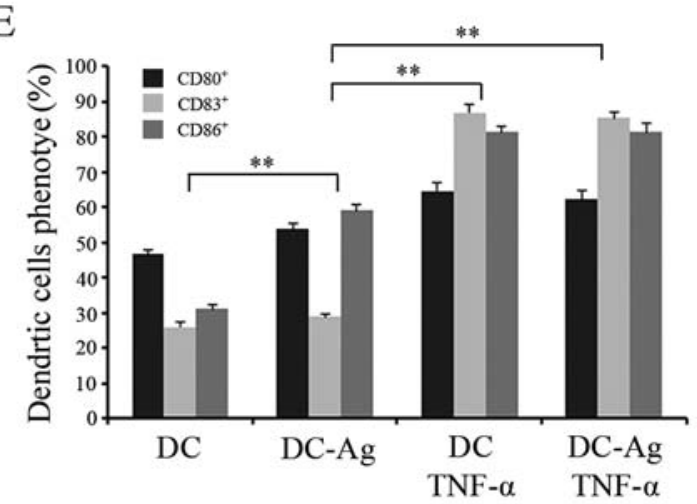

Figure 1. Morphological characterization and phenotypical analysis of DC maturation in vitro. (A) PBMCs on day 1 and DCs cultured in presence of GM-CSF, IL-4, or TNF- $\alpha$ for 7 days were plated on glass coverslips, fixed and stained for actin filaments with phallodin-TRITC (right). Cells were imaged using DIC microscopy and fluorescence for visualization of the actin filaments (right). Arrowheads show representatives PBMC cells (left) and arrows indicate distinctive features of a mature DC cell after culture in a cytokine-enriched media for 7 days. Scale bars, $20 \mu \mathrm{m}$. (B) DCs cultured in a cytokine-enriched media were incubated with CD80-PE, CD83-APC and CD86-FITC antibodies and the phenotypes of these cells were analyzed by FCM as described in Materials and methods. The figure shows the FCM data for the CD86 expression after 3 (left) and 7 days (right) in culture. The quantification of CD80, CD83 and CD86 expression for 3 and 7 days in culture is given as mean \pm SE from three independent experiments. ${ }^{* *} \mathrm{P}<0.01$ (C). (D) FCM analysis of CD86 expression after 7 days in culture on DC (top left), DC-Ag cells (top right) and with addition of TNF- $\alpha$ (bottom) in the culture media. (E) The quantification of CD80, CD83 and CD86 expression from the FCM data is given as mean \pm SE from three independent experiments. ${ }^{* *} \mathrm{P}<0.01$.

cytotoxic T-lymphocytes with the characteristic $\mathrm{CD} 3^{+} \mathrm{CD} 56^{+}$ phenotype. In order to generate CIK cells in vitro, the nonadherent PBMCs were separated and cultured in the presence of interferon- $\gamma$ (IFN- $\gamma)$, CD3 monoclonal antibody and IL-2 among other cytokines. After the 7th day in culture, cell morphology indicated that CIK cells had acquired the mature phenotype (Fig. 2A). At day 7, the cell area was on average five times bigger than at day 1 in culture (data no shown) depicting irregular shape. Multiple cells formed clusters and gathered into cell aggregates (arrows in Fig. 2A, left panel). Interestingly, we noted that the actin cytoskeleton of these mature CIK cells attained a polarized distribution (Fig. 2A, middle and right panels).

Next, we analysed the proportion of cells expressing $\mathrm{CD}^{+}{ }^{+} \mathrm{CD}^{2} 6^{+}, \mathrm{CD}^{+}{ }^{+} \mathrm{CD} 8^{+}, \mathrm{CD}^{+}$and $\mathrm{CD}^{+}$in the cell population that had grown in the cytokine-enriched media. As shown in Fig. $2 \mathrm{~B}$ and $\mathrm{C}$, the proportion of $\mathrm{CD}^{+}{ }^{+} \mathrm{CD} 56^{+}$and $\mathrm{CD} 3{ }^{+} \mathrm{CD} 8^{+}$ cells increased on day 15 compared with day $7(\mathrm{P}<0.01)$.

It has been reported that the CIK cells could interact with DCs resulting in an increment of CIK cytotoxic and cytolytic activities against tumor cells. To examine if this applies to our CIK in the presence of DC-Ag, we further examined whether these DC-Ag cells could expand the proportion of mature $\mathrm{CD}^{+}{ }^{+} \mathrm{CD} 56^{+} \mathrm{CIK}$ cells in culture. CIK and DC-CIK or DC-Ag-CIK phenotypes were assayed with fluorescenceactivated cell sorting analyses. On day 15 , the proportion of $\mathrm{CD}^{+}{ }^{+} \mathrm{DD}^{2} 6^{+}$and $\mathrm{CD}^{+}{ }^{+} \mathrm{CD} 8^{+}$cells were higher when the CIK cells were co-cultured with DC-Ag cells $(\mathrm{P}<0.01)$. No obvious differences were found when DC-CIK cells were compared with CIK cells $(\mathrm{P}>0.05)$. There were no significant differences for the population of $\mathrm{CD}^{+}$and $\mathrm{CD} 8^{+}$cells compared between the other groups $(\mathrm{P}>0.05)$ (Fig. 2D and $\mathrm{E})$. 
A

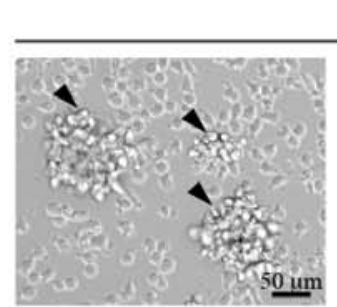

CIK 7 Days in vitro
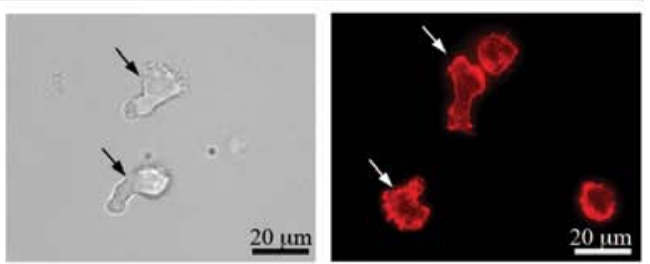

B

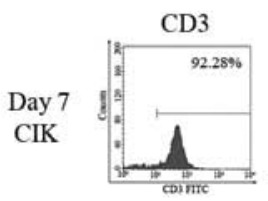

CD8
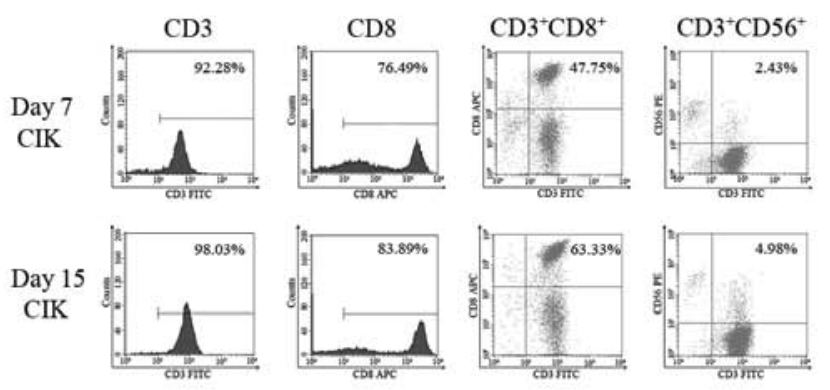

C

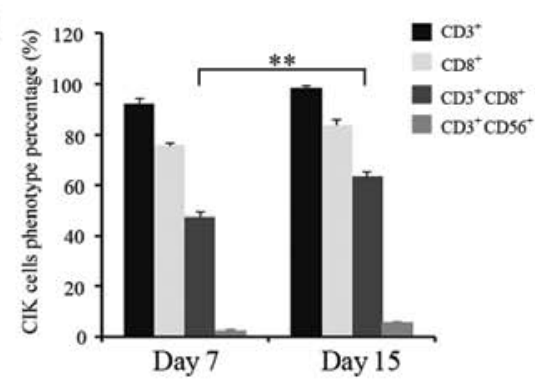

D
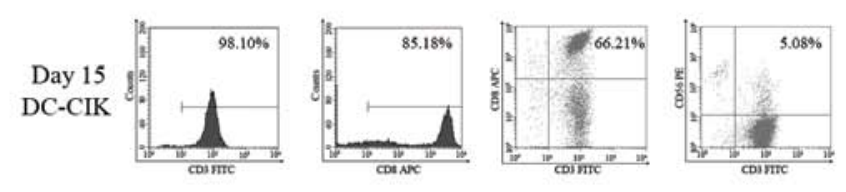

$\mathrm{E}$

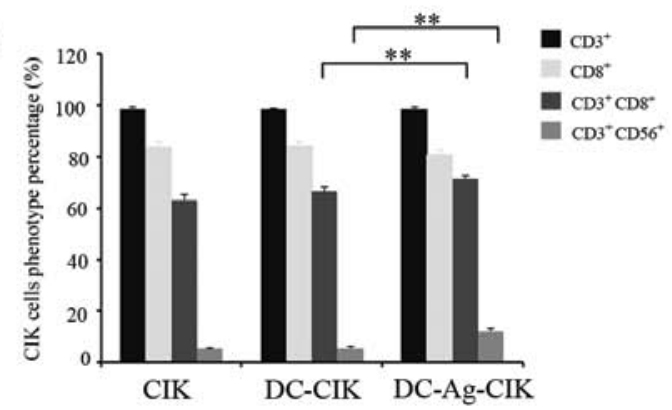

Figure 2. Morphological characterization and phenotypical analysis of CIK cell maturation in vitro. (A) CIK cells from non-adherent PBMCs cultured in the presence of IFN- $\gamma$ and IL-2, CD3 monoclonal antibody for 7 days was plated on glass coverslips, fixed and stained for actin filaments with phalloidin-TRITC (right). Cells were imaged using DIC microscopy and fluorescence for visualization of the actin filaments (right). Arrowheads indicate the cell clusters typical of differentiated CIK cell culture (left) and arrows indicate distinctive features of a mature CIK cell after culturing in a cytokine-enriched media for 7 days. (B) CIK cells cultured for 7 or 15 days were incubated with CD3-FITC, CD8-APC and CD56-PE antibodies. The phenotypes of these cells were analyzed by FCM as described in Materials and methods. The image showed the FCM data for the $\mathrm{CD} 3{ }^{+} \mathrm{CD} 56^{+}$expression after 7 (left) and 15 days (right) in culture. The quantification of $\mathrm{CD}^{+}, \mathrm{CD}^{+}, \mathrm{CD}^{+} \mathrm{CD}^{+}$and $\mathrm{CD}^{+} \mathrm{CD}^{+} 6^{+}$expression for 7 and 15 days in culture is given as mean $\pm \mathrm{SE}$ from three independent experiments. ${ }^{* *} \mathrm{P}<0.01$ (C). (D) FCM analysis of $\mathrm{CD}^{+} \mathrm{CD}^{2} 6^{+}$expression after 15 days in culture on CIK (left), CIK co-culture with DC (DC-CIK) (middle) and CIK co-culture with antigen loaded DC (Ag-DC-CIK) (right). (E) The quantification of $\mathrm{CD}^{+}, \mathrm{CD}^{+}, \mathrm{CD}^{+} \mathrm{CD}^{+}$and $\mathrm{CD}^{+} \mathrm{CD}^{+} 6^{+}$expression from the $\mathrm{FCM}$ data is given as mean $\pm \mathrm{SE}$ from three independent experiments. ${ }^{* *} \mathrm{P}<0.01$.

The results stated above showed the $\mathrm{CD} 3{ }^{+} \mathrm{CD} 56^{+} \mathrm{CIK}$ cell proportion increases in the T-lymphocyte population when they are co-cultured with DC-Ag cells.

Proliferation of CIK cells and cytokine production. We found that CIK cells in culture proliferated in a non-exponential manner, especially from day 9 onwards (Fig. 3A). To examine whether DCs or DC-Ag affected CIK proliferation, CIK cells were co-cultured with DCs or DC-Ag from day 7 to 15 and the cell number per $\mathrm{ml}$ of medium was quantified everyday in the different groups (Fig. 3A). After 15 days in culture the absolute cell number had significantly $(\mathrm{P}<0.01)$ increased $5.36 \pm 1.58,8.37 \pm 2.19$ and 9.38 \pm 1.97 -fold in CIK, DC-CIK and DC-Ag-CIK, respectively (Fig. 3A).

To investigate a possible cause for the differences observed on CIK cell proliferation, we determined the level of two cytokines that have been shown to play a role in the proliferation of these cells (IL-6 and IL-10). IL-6 was selected because it was shown to have stimulatory effect on CIK proliferation and IL-10 because it has inhibitory effect on primary alloreactive
T-cell responses $(27,28)$. The supernatants from CIK and DC-CIK or DC-Ag-CIK cultures were collected on day 15 and the specific cytokines were quantified by using a Cytometric Bead Array Human Th1/Th2 cytokine kit. As shown in Fig. 3B and C, IL-6 levels increased 3-fold when CIK cells were grown in the presence of DC cells or DC-Ag $(1.65 \pm 0.13 \mathrm{pg} /$ $\mathrm{ml})$ and $(1.78 \pm 0.28 \mathrm{pg} / \mathrm{ml})$, respectively, compared with levels of secreted IL- 6 in CIK culture alone $(0.57 \pm 0.16 \mathrm{pg} / \mathrm{ml})$ $(\mathrm{P}<0.01)$. In contrast, secreted IL-10 level was two-fold lower in DC-Ag-CIK cell co-culture (Fig. 3C) compared with CIK or CIK co-cultured with DC cells (Fig. 3B and D). These results indicate that CIK cells increased proliferation in the presence of DCs and DC-Ag might be a consequence of the combination of a low level of the inhibitory IL-10 and elevation of the stimulatory IL-6 levels in these co-culture conditions.

Cytotoxicity assay. Having shown that the DC-Ag cell enhanced CIK proliferation and differentiation in vitro (Figs. 1 and 2) we next tested whether these processes translated to an increment in CIK cytotoxic activity on RB-Y79 cells (Fig. 4). 
A

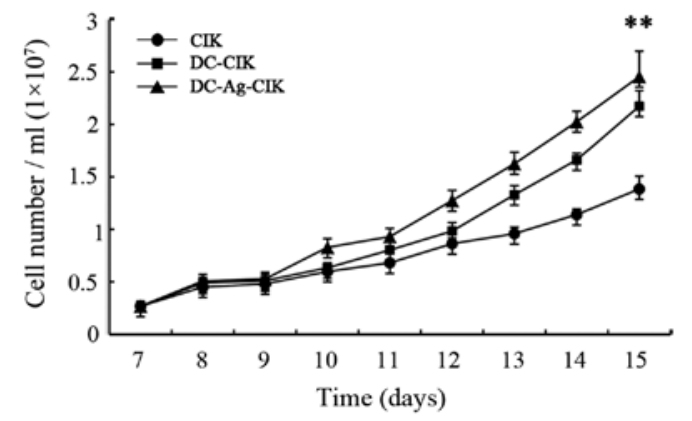

B
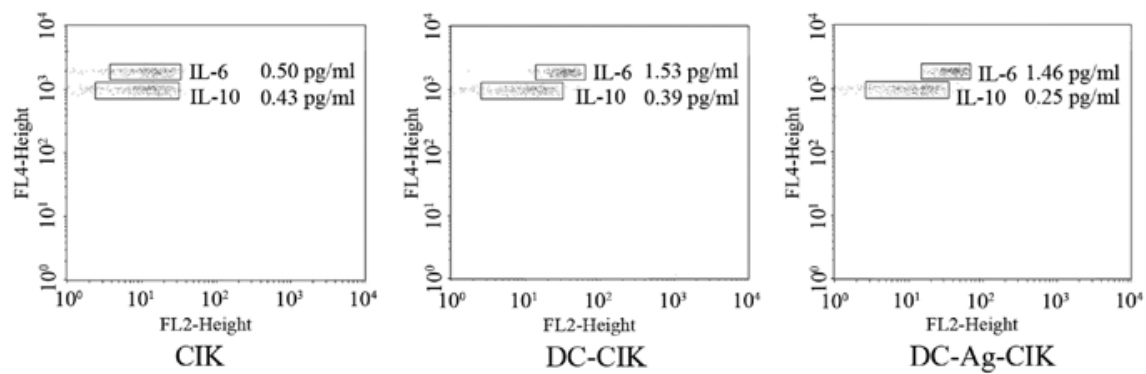

$\mathrm{C}$

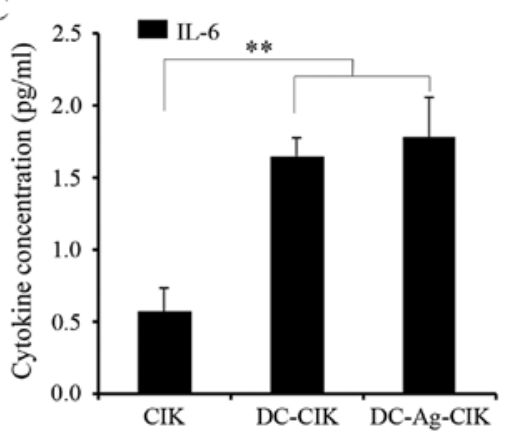

$\mathrm{D}$

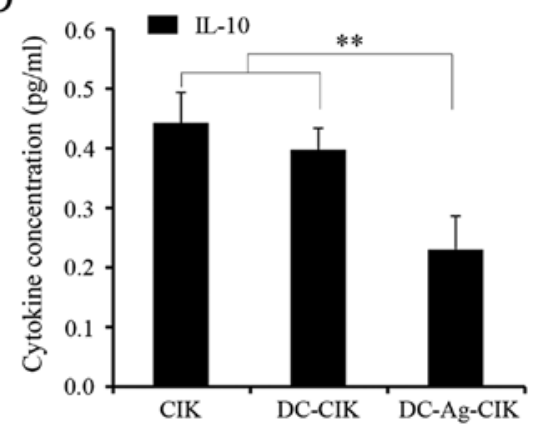

Figure 3. Proliferation of CIK cells and cytokine production. (A) Quantification of the number of CIK cells cultured from 7 to 15 days in CIK alone (•), CIK co-culture with DC cells ( $\mathbf{\square})$ and CIK co-cultured with antigen loaded DC cells ( $\mathbf{\Delta})$. (B-D) FCM analysis of IL-6 and IL-10 cytokines secreted in the CIK, DC-CIK and DC-Ag-CIK media culture. Supernatants from CIK alone, CIK co-culture with DC cells (CIK-DC) and CIK co-cultured with antigen loaded DC cells (DC-Ag-CIK) were collected and the levels of IL-6 (C) and IL-10 (D) cytokines were determined by Cytometric Bead Array Human Th1/Th2 cytokine kit II (B). Values are given as mean \pm SE from three independent experiments. ${ }^{* *} \mathrm{P}<0.01$.

CIK cells were co-cultured either with autologous nonpulsed DCs or tumor lysate-pulsed DCs (DC-Ag) for 7 days starting on culture day 7 and tested for cytotoxicity against RB-Y79 cell line at day 14 . The cytotoxicity was assayed by FCM using specific dyes for labeling viable and dead cells. The results, shown in Fig. 4, are expressed as the proportion of cells that are dead relative to the total number of cancer cells in the culture, minus the natural cell death observed in each condition.

As shown in Fig. 4A, CIK cells generated by the cytokine-enriched media killed $\sim 6 \%$ of the RB-Y79 cells after co-culturing for $24 \mathrm{~h}$ at a ratio of 10:1 [effector cells:target cells (E:T)]. The cytotoxicity doubled when the CIK had been co-cultured with the DCs for 7 days. Moreover, the CIK cytotoxicity was significantly higher when they were in the presence of DC-Ag $(\mathrm{P}<0.001)$. Higher E:T ratios resulted in significantly higher cytotoxicity $(\mathrm{P}<0.01)$ throughout the experimental conditions. This observation suggests that the effects are unlikely to result from unspecific influences, other than the killer cells.
To rule out the possibility that the cytotoxicity was the result of some unidentified agent present in the cell media rather than by the CIK cell activity, the cytotoxic of the assay was performed on RB-Y79 cells incubating the CIK with conditioned media taken from DC or DC-Ag cultures instead of with the DCs or DC-Ag. No significant difference was found on the CIK cytotoxic activity on RB-Y79 cells between the 3 treatments (Fig. 4B) ( $\mathrm{P}>0.05)$. These observations suggested that the enhancement of cytotoxic activity on tumor cells could be at least in part explained by the direct interaction of CIK cells with mature DC rather than by activity of the components we added to the culture media.

CIK cells have been described as a highly efficient cytotoxic effector capable of lysing tumor cell targets by a mechanism that is non-MHC restricted. Because of this, we explored whether CIK cells (mature and activated with DC and DC-Ag) have specific cytotoxic activity on RB cell lines or if they also targeted normal retinal cells. We set up the cytotoxic assay as described above and we determined the CIK cytotoxic activity on hTERT-RPE1 cells. Surprisingly, no significant cytotox- 
A

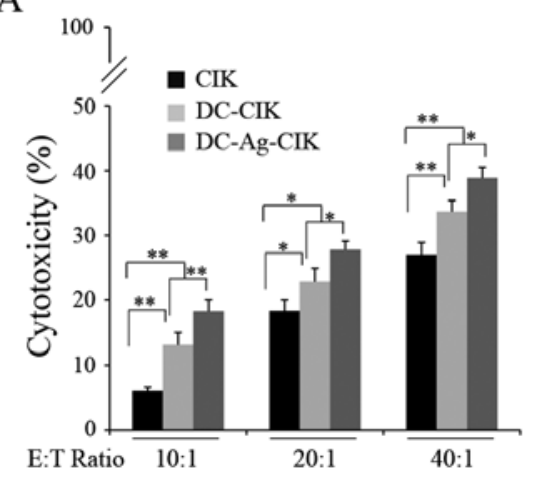

B

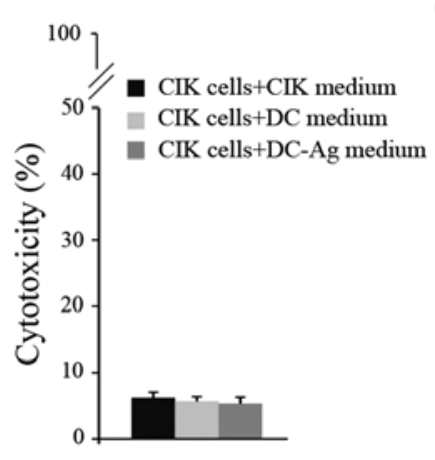

$\mathrm{C}$

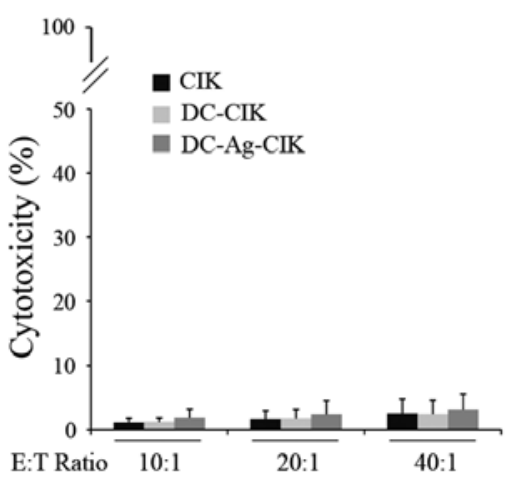

Figure 4. Cytotoxicity in vitro of the effector cells. (A-C) CIK, DC-CIK or DC-Ag-CIK effector cells were cultured for 15 days. The resulting effector cell populations were used to examine cytotoxicity on RB-Y79 (A) and RPE1 cells (C). Target cells were labeled with CFSE and incubated for $24 \mathrm{~h}$ with effector cells at E:T ratios of 10:1, 20:1 and 40:1. Dead cells labeled with CFSE/PI were detected by FCM. (B) Cytotoxicity of CIK cells against RB-Y79 cells cultured in medium from CIK culture, DCs culture medium or DC-Ag culture medium. Data are given as mean \pm SE from three independent experiments. ${ }^{* *} \mathrm{P}<0.01$.

A

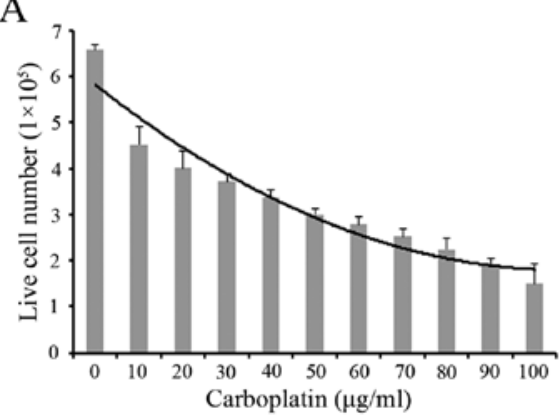

$\mathrm{C}$

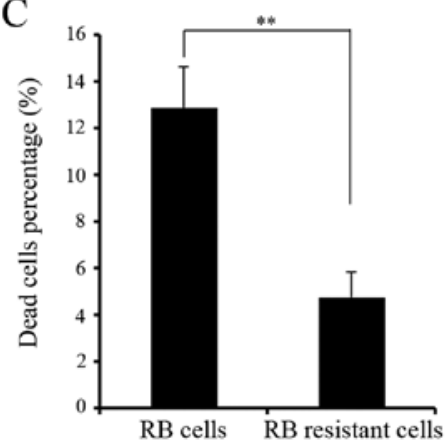

B
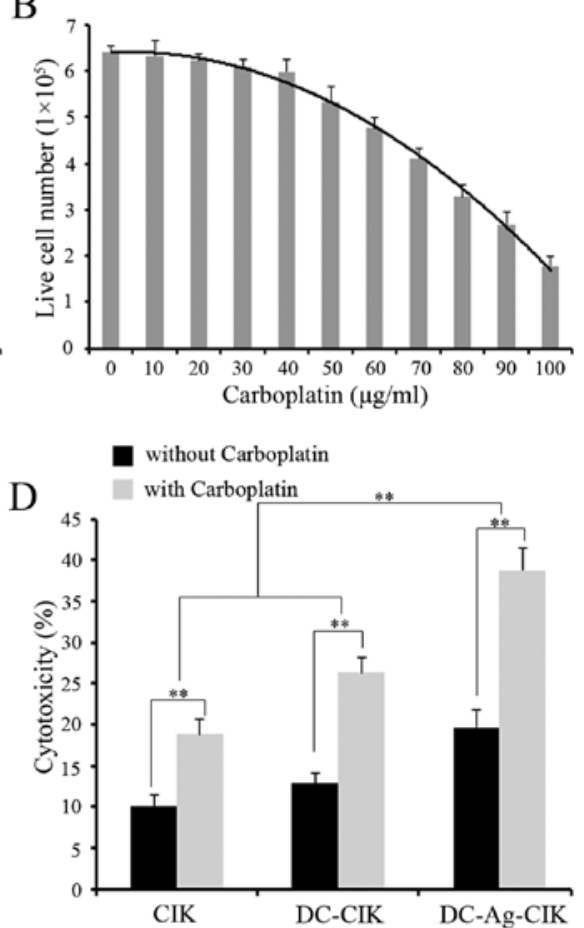

Figure 5. In vitro cytotoxicity of immunotherapy on carboplatin-resistant cells in combination with carboplatin. (A and B) RB-Y79 cells (A) and RB-Y79 carboplatin resistance cells (RB-R) (B) were cultured in the presence of 10, 20, 40, 50, 60, 70, 80, 90 and $100 \mu \mathrm{g} / \mathrm{ml}$ carboplatin respectively for $24 \mathrm{~h}$. Cell numbers were counted in the culture using Counter Star with the software Automated Cell Counter. Data are given as mean \pm SE from three independent experiments. (C) RB-Y79 cells and RB-R cells were cultured with $40 \mu \mathrm{g} / \mathrm{ml}$ carboplatin for $24 \mathrm{~h}$ and dead cells were measure by PI/CFSE staining. Data are given as mean \pm SE from three independent experiments. ${ }^{* *} \mathrm{P}<0.01$. (D) FCM analysis of cytotoxic activity of CIK cells, DC-CIK cells and DC-Ag-CIK cells on RB-R cells in combination with carboplatin. Data are given as mean \pm SE from three independent experiments. ** $\mathrm{P}<0.01$.

icity was detected on hTERT-RPE1 cells in any of the assayed groups. The CIK cytotoxic activity on these cells was $1.13 \%$ at an E:T ratio of 10:1 (Fig. 4C).

Cytotoxicity assay for carboplatin-resistant RB cells. After establishing the RB-Y79 carboplatin-resistant cell line, cells were tested for their ability to survive and proliferate in a media containing carboplatin. Resistant and sensitive RB cells were cultured with increasing dosis of carboplatin and the cell survival was analysed after $24 \mathrm{~h}$. The result showed that the number of live RB-sensitive cells decreased significantly as the carboplatin concentration increased. In contrast, the number of RB-resistant living cells was not significantly affected until a dose of $50 \mu \mathrm{g} / \mathrm{ml}$ of carboplatin. However, RB-resistant survival cells gradually decreased with $50 \mu \mathrm{g} / \mathrm{ml}$ or higher drug concentration (Fig. 5A and B). Given this ability of the RB-resistant cells to survive at $40 \mu \mathrm{g} / \mathrm{ml}$ carboplatin, we named it RB-R cells.

In order to prove that RB-R cells are resistant to carboplatin, $40 \mu \mathrm{g} / \mathrm{ml}$ of carboplatin was used on RB-sensitive cells 
and RB-R cells. The result showed that the ability to survive carboplatin was more than two-fold increased in RB-R cells compared to the sensitive RB cells (Fig. 5C).

We have shown that CIK, DC-CIK and DC-Ag-CIK cells had a strong cytotoxic activity on normal RB cells, we wanted to test whether the effector cells could kill RB-R cells efficiently. CIK, DC-CIK and DC-Ag-CIK cells were used after 7 days co-culture on day 14 and tested for cytotoxicity against RB-R cell line at $40 \mu \mathrm{g} / \mathrm{ml}$. The cytotoxicity was assayed by FCM using specific dyes for labeling viable and dead cells. DC-Ag-CIK cells generated by the cytokine-enriched media killed $\sim 20 \%$ of the RB-R cells after co-culturing for $24 \mathrm{~h}$ at an E:T ratio of 20:1 (Fig. 5D). The cytotoxicity of DC-Ag-CIK cells against RB-R cells were higher than DC-CIK and CIK cells $(\mathrm{P}<0.01)$, but there was no significant difference between DC-CIK and CIK cells ( $\mathrm{P}>0.05)$. To test the combined effect of carboplatin, RB-R cells co-cultured with effector cells were incubated with $40 \mu \mathrm{g} / \mathrm{ml}$ carboplatin for $24 \mathrm{~h}$. The cytotoxicity of DC-Ag-CIK in combination with carboplatin showed the best antitumor activity compared with DC-CIK and CIK cells, and DC-CIK were higher than CIK cells $(\mathrm{P}<0.01)$. In all groups, the effector cells that were combined with carboplatin showed higher cytotoxicity than effector cells alone $(\mathrm{P}<0.01)$.

\section{Discussion}

In recent years, there has been growing interest in cancer immunotherapy. After reducing tumor burden, immunotherapy can be used as an adjunctive treatment that can effectively remove the residual tumor cells. Our study focused on the two principal players: the T-lymphocytes and the DCs. DCs are the most powerful antigen-presenting cells, with a capacity of antigen-presentation that is hundreds of times more efficient than B lymphocytes and macrophages (29). They can activate resting T-cells and induce the generation of antigen-specific cytotoxic T-lymphocytes, which are the initiators of the immune response (30). On the other hand, the CIK cells are a population of heterogeneous T-lymphocytes generated by the in vitro differentiation of mononuclear cells from the peripheral blood (31). CIK cells cultured with multiple cytokines including IL-2, CD3 monoclonal antibody and IFN- $\gamma$ and those that express the $\mathrm{CD} 3{ }^{+} \mathrm{CD} 56^{+}$have been shown to have a strong antitumor effect $(19,32,33)$. Among their advantages over other T-lymphocytes generated in vitro from peripheral blood such as LAK/NK cells, CIK cells are easy to generate in large numbers, readily expandable from cancer patients (34), with minimal graft-versus host reaction (35) and the cytotoxic activity is not restricted to MHC $(36,37)$. Immunotherapy has been used for treating a variety of adult solid tumors such as liver cancer, gastric carcinoma, melanoma, and renal carcinoma with good efficacy $(32,38-41)$. For pediatric solid tumors, some studies have shown that CIK treatment have a good antitumor activity in vitro on lymphoma, osteosarcoma, Ewing's family tumors and neuroblastoma $(42,43)$, but only few studies have been reported on the treatment of children because of a lower incidence of pediatric tumors and due to ethical issues.

One practical strategy for improving the effectiveness of immunotherapy relies on the fact that enhanced cytotoxicity is gained when CIK cells are co-culture with DCs (44-46) and this effect is even stronger when CIK are co-cultured with tumor antigen pulsed DCs $(20,47)$. Combining DCs pulsed with $\mathrm{Ag}$ (which exhibit unique antigen-presenting function) with CIK cells (which have strong antitumor activity) would probably cause a double antitumor effect. In pediatric oncology a few tumor-associated antigens have been identified (48). This problem is solved in part by the use of the whole-tumor lysed as a source of tumor-antigens for DC-loading. This would induce a stronger immune response than that obtained by pulsing DCs with a single or perhaps several defined tumor peptides (49). In addition, with this approach, there is no need for revealing the nature of the tumor-associated antigen and its epitope. Therefore, for our study we used a retinoblastoma (RB) cell line (RB-Y79 cells) lysates as tumor antigen to load DCs.

To develop an effective cancer therapy there are at least three important aspects we have to achieve: one is obtaining enough effector cell numbers, two, producing effector cells with a high cytotoxic activity against tumor cells and three, generating effector cells with specific cytotoxicity for the target cell.

In PBMCs the normal proportion of $\mathrm{CD}^{+} \mathrm{CD}^{+} 6^{+}$cells is low $(1-5 \%)$. We have been able to obtain up to $40 \%$ of $\mathrm{CD}^{+}{ }^{+} \mathrm{CD} 56^{+}$cells under certain experimental conditions (data not shown). This very high proportion will make it difficult to discern what might be modest effects of the Ag or of co-culture with DC cells. To prevent this, we used a cytokine-enriched media to achieve a basal level of maturation that allowed us to detect slight differences of DC-Ag on CIK differentiation. In fact, the CIK proportion after 15 days in culture increased by $\sim 300 \%$ of $\mathrm{CD}^{+} \mathrm{CD}^{2} 6^{+}$proportion in the presence of the DC-Ag cells.

In our study, the DC-Ag-CIK cells were substantially more cytotoxic against RB cells than DC-CIK and CIK cells alone. This increment in the CIK cytotoxic activity when co-cultured with DC pulsed with the Ag is probably due to both an increment of the proportion of T-lymphocyte expressing $\mathrm{CD}^{+} \mathrm{CD}^{2} 6^{+}$plus a larger number of cells actively differentiating and proliferating. The increment in cell number could be explained by the fact that the secreted IL- 6 is higher in the co-cultured DC-Ag-CIK inducing cells to proliferate. IL-6 is a pleiotropic cytokine secreted by DCs, T-lymphocytes and macrophages, which has been shown to support the growth of T- and B-lymphocytes in vitro (27). Another possible explanation could be that IL- 6 triggers IL- 2 secretion which in turn can promote autocrine CIK proliferation (50). Accordingly, a decrease in the level of secreted IL-10 could promote proliferation. It has been reported that IL-10 downregulates CD80 and CD86 expression at the DC surface therefore having an inhibitory effect on alloreactive T-cell responses (28). On the other hand, IL-10 has been involved in decreased secretion of IL-12 and IFN- $\gamma$ which are the cytokines reported to promote lymphocyte proliferation (28). Our results are consistent with IL-6 and IL-10 playing a role in the observed proliferation of CIK cells. However, the extent to which this may account for the net effects seen needs to be addressed.

Regardless whether the effector cells were co-cultured with DCs or Ag pulsed DCs or even CIK alone, there was no significant cytotoxicity on normal retina cells. This fact can not be explained by the presence of the $\mathrm{Ag}$ or the DC func- 
tion itself since the CIK themselves failed to kill the normal retina cells. This provides preliminary evidence supporting the safety of this approach in the clinical practice for RB. It is also important to highlight that the enhanced cytotoxicity, the high proliferation ratio, the increment in differentiation of the $\mathrm{CD}^{+} \mathrm{CD}^{2} 6^{+}$cells and the specificity of the cytotoxicity on tumor cells are not due to components or cytokines added to the culture media. Furthermore, those improve features of CIKs are rather the direct result of the DCs and the DC-Ag cells on the CIK maturation and function. Lin et al (51) showed in vitro that the addition of IL- 6 in the culture medium could increase $\mathrm{CD}^{+}{ }^{+} \mathrm{CD} 56^{+} \mathrm{T}$-lymphocytes and therefore enhances proliferation and cytotoxicity of effector cells. In light of this report, it would be useful to examine whether the CIK-Ag-DC cells in this study are comparable in cytotoxic activity against RB cells to the CIK-IL-6 cells described by Lin et al (51).

Finally, we present substantial evidence of effective cytotoxicity of the combination of CIK with DC-Ag on tumor RB cells. Albeit our CIK active proportion is relatively low under our experimental conditions, once the DC cells are in the blood stream they could still activate the naïve T-lymphocytes. In this event, administration of the complex CIK-Ag-DC can have advantages over other immunotherapies. In fact, it has been reported that injected DC-CIK in the body exert their action for $\leq 40$ days. The efficacy of this therapy has yet to be demonstrated in animal paradigms. This study demonstrates for the first time that an immunotherapy-based approach using CIK cells could be effective to treat $\mathrm{RB}$, mainly because it has selective effect on carboplatin-resistant retinoblastoma cells. Thus highly efficient immunotherapy based on DC-Ag-CIK cells is a potentially effective and safe means of treating $\mathrm{RB}$ especially for patients where traditional chemical therapy has failed.

\section{Acknowledgements}

We are grateful to Dr Cristian Acosta and Dr Yue Sun for insightful comments on the manuscript. We thank Poten Biomedical Co. (Shenzhen, China) for providing technical support and Guillaume Charras for Live-ActGFP plasmid. This study was supported by both Shenzhen Development and Reform Commission [grants no. (2011)1680] and the Capital Clinical Features Applied Research Plan (grants no. Z121107001012055). P.K., J.L, H.W, X.C and T.L. are supported in part by Poten Biomedical Institute for Cancer Immunotherapy.

\section{References}

1. Dimaras H, Kimani K, Dimba EA, et al: Retinoblastoma. Lancet 379: 1436-1446, 2012.

2. Bai S, Ren R, Li B, et al: Delay in the diagnosis of retinoblastoma in China. Acta Ophthalmol 89: e72-74, 2011.

3. MacCarthy A, Draper GJ, Steliarova-Foucher E and Kingston JE: Retinoblastoma incidence and survival in European children (1978-1997). Report from the Automated Childhood Cancer Information System project. Eur J Cancer 42: 2092-2102, 2006.

4. Gallie BL, Zhao J, Vandezande K, White A and Chan HS: Global issues and opportunities for optimized retinoblastoma care. Pediatr Blood Cancer 49: 1083-1090, 2007.

5. Houston SK, Murray TG, Wolfe SQ and Fernandes CE: Current update on retinoblastoma. Int Ophthalmol Clin 51: 77-91, 2011.
6. Radhakrishnan V, Kashyap S, Pushker N, et al: Outcome, pathologic findings and compliance in orbital retinoblastoma (International Retinoblastoma Staging System stage III) treated with neoadjuvant chemotherapy: a prospective study. Ophthalmology 119: 1470-1477, 2012.

7. Pica A, Moeckli R, Balmer A, et al: Preliminary experience in treatment of papillary and macular retinoblastoma: evaluation of local control and local complications after treatment with linear accelerator-based stereotactic radiotherapy with micromultileaf collimator as second-line or salvage treatment after chemotherapy. Int J Radiat Oncol Biol Phys 81: 1380-1386, 2011.

8. Wilson MW, Fraga $\mathrm{CH}$, Rodriguez-Galindo $\mathrm{C}$, Hagedorn $\mathrm{N}$, Leggas ML and Stewart C: Expression of the multi-drug resistance proteins and the pregnane $\mathrm{X}$ receptor in treated and untreated retinoblastoma. Curr Eye Res 34: 386-394, 2009.

9. Rutella S, Iudicone P, Bonanno G, et al: Adoptive immunotherapy with cytokine-induced killer cells generated with a new good manufacturing practice-grade protocol. Cytotherapy 14: 841-850, 2012.

10. Campoli M, Ferris R, Ferrone S and Wang X: Immunotherapy of malignant disease with tumor antigen-specific monoclonal antibodies. Clin Cancer Res 16: 11-20, 2010.

11. Leffers N, Daemen T, Helfrich W, et al: Antigen-specific active immunotherapy for ovarian cancer. Cochrane Database Syst Rev CD007287, 2010.

12. Palucka K, Ueno H, Fay J and Banchereau J: Dendritic cells and immunity against cancer. J Intern Med 269: 64-73, 2011.

13. Cella M, Sallusto F and Lanzavecchia A: Origin, maturation and antigen presenting function of dendritic cells. Curr Opin Immunol 9: 10-16, 1997.

14. de Vleeschouwer S, Rapp M, Sorg RV, et al: Dendritic cell vaccination in patients with malignant gliomas: current status and future directions. Neurosurgery 59: 988-999, 2006.

15. Schnurr M, Galambos P, Scholz C, et al: Tumor cell lysate-pulsed human dendritic cells induce a T-cell response against pancreatic carcinoma cells: an in vitro model for the assessment of tumor vaccines. Cancer Res 61: 6445-6450, 2001.

16. Wu YG, Wu GZ, Wang L, Zhang YY, Li Z and Li DC: Tumor cell lysate-pulsed dendritic cells induce a $T$ cell response against colon cancer in vitro and in vivo. Med Oncol 27: 736-742, 2010.

17. Mesiano G, Todorovic M, Gammaitoni L, et al: Cytokineinduced killer (CIK) cells as feasible and effective adoptive immunotherapy for the treatment of solid tumors. Expert Opin Biol Ther 12: 673-684, 2012.

18. Shi L, Zhou Q, Wu J, et al: Efficacy of adjuvant immunotherapy with cytokine-induced killer cells in patients with locally advanced gastric cancer. Cancer Immunol Immunother 61: 2251-2259, 2012.

19. Zhang J, Zhu L, Wei J, et al: The effects of cytokine-induced killer cells for the treatment of patients with solid tumors: a clinical retrospective study. J Cancer Res Clin Oncol 138: 1057-1062, 2012.

20. Zhan HL, Gao X, Pu XY, et al: A randomized controlled trial of postoperative tumor lysate-pulsed dendritic cells and cytokineinduced killer cells immunotherapy in patients with localized and locally advanced renal cell carcinoma. Chin Med J (Engl) 125: 3771-3777, 2012.

21. Yang L, Ren B, Li H, et al: Enhanced antitumor effects of DC-activated CIKs to chemotherapy treatment in a single cohort of advanced non-small-cell lung cancer patients. Cancer Immunol Immunother: Jun 29, 2012 (Epub ahead of print).

22. Zhong R, Teng J, Han B and Zhong H: Dendritic cells combining with cytokine-induced killer cells synergize chemotherapy in patients with late-stage non-small cell lung cancer. Cancer Immunol Immunother 60: 1497-1502, 2011.

23. Komada Y, Zhang SL, Zhou YW, et al: Cellular immunosuppression in children with acute lymphoblastic leukemia: effect of consolidation chemotherapy. Cancer Immunol Immunother 35: 271-276, 1992.

24. Mackall CL: T-cell immunodeficiency following cytotoxic antineoplastic therapy: a review. Stem Cells 18: 10-18, 2000.

25. Schmidt-Wolf IG, Lefterova P, Mehta BA, et al: Phenotypic characterization and identification of effector cells involved in tumor cell recognition of cytokine-induced killer cells. Exp Hematol 21: 1673-1679, 1993.

26. Pletinckx K, Stijlemans B, Pavlovic V, et al: Similar inflammatory DC maturation signatures induced by TNF or Trypanosoma brucei antigens instruct default Th2-cell responses. Eur J Immunol 41: 3479-3494, 2011. 
27. Puri RK and Leland P: Systemic administration of recombinant interleukin-6 in mice induces proliferation of lymphoid cells in vivo. Lymphokine Cytokine Res 11: 133-139, 1992.

28. Buelens C, Willems F, Delvaux A, et al: Interleukin-10 differentially regulates $\mathrm{B} 7-1(\mathrm{CD} 80)$ and $\mathrm{B} 7-2$ (CD86) expression on human peripheral blood dendritic cells. Eur J Immunol 25 : 2668-2672, 1995.

29. Kulkarni AB, Mullbacher A and Blanden RV: Functional analysis of macrophages, B cells and splenic dendritic cells as antigen-presenting cells in West Nile virus-specific murine T lymphocyte proliferation. Immunol Cell Biol 69: 71-80, 1991.

30. Hasel T, Yoshimura R, Wada S and Chargui J: Dendritic cells, generated in vitro, are immunocompetent and very useful in the induction of specific cytotoxic T lymphocyte activity. Transplant Proc 33: 3814-3815, 2001

31. Lu PH and Negrin RS: A novel population of expanded human $\mathrm{CD}^{+}{ }^{+} \mathrm{CD} 56^{+}$cells derived from $\mathrm{T}$ cells with potent in vivo antitumor activity in mice with severe combined immunodeficiency. J Immunol 153: 1687-1696, 1994.

32. Wang FS, Liu MX, Zhang B, et al: Antitumor activities of human autologous cytokine-induced killer (CIK) cells against hepatocellular carcinoma cells in vitro and in vivo. World J Gastroenterol 8: 464-468, 2002.

33. Ma Y, Zhang Z, Tang L, et al: Cytokine-induced killer cells in the treatment of patients with solid carcinomas: a systematic review and pooled analysis. Cytotherapy 14: 483-493, 2012.

34. Alvarnas JC, Linn YC, Hope EG and Negrin RS: Expansion of cytotoxic $\mathrm{CD}^{+} \mathrm{CD}^{+} 6^{+}$cells from peripheral blood progenitor cells of patients undergoing autologous hematopoietic cell transplantation. Biol Blood Marrow Transplant 7: 216-222, 2001

35. Baker J, Verneris MR, Ito M, Shizuru JA and Negrin RS Expansion of cytolytic CD8(+) natural killer T cells with limited capacity for graft-versus-host disease induction due to interferon gamma production. Blood 97: 2923-2931, 2001.

36. Schmidt-Wolf IG, Negrin RS, Kiem HP, Blume KG and Weissman IL: Use of a SCID mouse/human lymphoma model to evaluate cytokine-induced killer cells with potent antitumor cell activity. J Exp Med 174: 139-149, 1991.

37. Leemhuis T, Wells S, Scheffold C, Edinger $M$ and Negrin RS A phase I trial of autologous cytokine-induced killer cells for the treatment of relapsed Hodgkin disease and non-Hodgkin lymphoma. Biol Blood Marrow Transplant 11: 181-187, 2005.

38. Zhang YS, Yuan FJ, Jia GF, et al: CIK cells from patients with HCC possess strong cytotoxicity to multidrug-resistant cell line Bel-7402/R. World J Gastroenterol 11: 3339-3345, 2005.

39. Jiang JT, Shen YP, Wu CP, et al: Increasing the frequency of CIK cells adoptive immunotherapy may decrease risk of death in gastric cancer patients. World J Gastroenterol 16: 6155-6162, 2010.
40. Chen J, Huang X, Huang G, Chen Y, Chen L and Song H Preconditioning chemotherapy with cisplatin enhances the antitumor activity of cytokine-induced killer cells in a murine melanoma model. Cancer Biother Radiopharm 27: 210-220, 2012.

41. Su X, Zhang L, Jin L, et al: Immunotherapy with cytokineinduced killer cells in metastatic renal cell carcinoma. Cancer Biother Radiopharm 25: 465-470, 2010.

42. Hongeng S, Petvises S, Worapongpaiboon S, Rerkamnuaychoke B, Pakakasama S and Jootar S: Generation of $\mathrm{CD}^{+} \mathrm{CD}^{+} 6^{+}$cytokine-induced killer cells and their in vitro cytotoxicity against pediatric cancer cells. Int J Hematol 77: 175-179, 2003.

43. Verneris MR, Arshi A, Edinger M, et al: Low levels of Her2/ neu expressed by Ewing's family tumor cell lines can redirect cytokine-induced killer cells. Clin Cancer Res 11: 4561-4570, 2005.

44. Wang QJ, Wang H, Pan K, et al: Comparative study on antitumor immune response of autologous cytokine-induced killer (CIK) cells, dendritic cells-CIK (DC-CIK) and semi-allogeneic DC-CIK. Chin J Cancer 29: 641-648, 2010.

45. Shi SB, Ma TH, Li CH and Tang XY: Effect of maintenance therapy with dendritic cells: cytokine-induced killer cells in patients with advanced non-small cell lung cancer. Tumori 98: 314-319, 2012.

46. Yang XJ, Huang JA, Lei W, Zhu YB and Zhang XG: Antitumor effects of cocultured dendritic cells and cytokine-induced killer cells on lung cancer in vitro and in vivo. Ai Zheng 25: 1329-1333, 2006.

47. Tan G, Zhang X, Feng H, Luo H and Wang Z: The therapeutic effect of cytokine-induced killer cells on pancreatic cancer enhanced by dendritic cells pulsed with K-ras mutant peptide. Clin Dev Immunol 2011: 649359, 2011.

48. Coughlin CM, Vance BA, Grupp SA and Vonderheide RH: RNA-transfected CD40-activated B cells induce functional T-cell responses against viral and tumor antigen targets: implications for pediatric immunotherapy. Blood 103: 2046-2054, 2004.

49. Yufeng D, Guocheng Z, Dongliang X, et al: Whole-tumorantigen-pulsed dendritic cells elicit cytotoxic T-cell response against pediatric nasopharyngeal carcinoma in vitro. Med Oncol 26: 78-85, 2009

50. Holsti MA and Raulet DH: IL-6 and IL-1 synergize to stimulate IL-2 production and proliferation of peripheral T cells. J Immunol 143: 2514-2519, 1989.

51. Lin G, Wang J, Lao X, et al: Interleukin-6 inhibits regulatory $\mathrm{T}$ cells and improves the proliferation and cytotoxic activity of cytokine-induced killer cells. J Immunother 35: 337-343, 2012. 